\title{
Kant, la teoría de la relatividad y la filosofía de la ciencia de comienzos del siglo XX
}

\author{
Álvaro J. Peláez Cedrés
}

\section{I}

\section{ntroducción}

En 1905 hizo su irrupción en la escena científica el artículo de Einstein "Zur Elektrodinamik bewegter Körper" ("Sobre la electrodinámica de los cuerpos en movimiento"). ${ }^{1}$ Este trabajo intentaba solucionar una dificultad que afrontaba la electrodinámica clásica en el contexto de la física newtoniana, a saber, que dicha teoría no podía satisfacer el principio de relatividad proclamado por el propio Newton, según el cual todos los marcos de referencia inerciales son físicamente equivalentes. Antes bien, al postular la existencia de fuerzas dependientes de la velocidad de los cuerpos sobre los cuales ellos actúan, e incluir en sus ecuaciones fundamentales una constante igual a la velocidad de la luz en el vacío, la electrodinámica presuponía la existencia de un marco de referencia privilegiado al cual dichas velocidades están referidas. Se entendía que ése era el marco en que reposa el éter, el medio que vibra con las ondas electromagnéticas. Pero ningún experimento permitía detectar la velocidad de la Tierra en el éter. ${ }^{2}$

Alentado por los escritos de Poincaré, Einstein se resolvió a postular la validez irrestricta del clásico principio de relatividad, extendiéndolo también a los fenómenos electromagnéticos. Al mismo tiempo, postuló la constancia de la velocidad de la luz en el vacío para un marco de referencia inercial y, por lo tanto, para todos. Es claro que esto fue posible gracias a que Einstein sometió a examen crítico tanto la variable tiempo que figura en las ecuaciones de la física, como la concepción del espacio, dominante desde la física newtoniana.

${ }^{1}$ Publicado originalmente en Annalen der Physik, vol. 17, pp. 891-921.

${ }^{2}$ Esta imposibilidad fue mostrada por el famoso experimento desarrollado por Michelson y Morley en 1887. 
Ahora bien, la filosofía, y en especial la epistemología de principios del siglo $\mathrm{XX}$, no ignoró los resultados de esta revolución en la teoría física. En efecto, varios filósofos y científicos de mentalidad filosófica reaccionaron de una manera notable a la nueva forma de concebir las relaciones en el mundo físico, no reconociendo dichos resultados como algo que le ocurría exclusivamente a la física, sino viendo las repercusiones profundas que dichos resultados podían tener para el contenido de la epistemología o la teoría del conocimiento. Entre estos intelectuales pueden destacarse al menos tres importantes: Moritz Schlick, Hans Reichenbach y Ernst Cassirer.

Como es más o menos bien conocido, Kant había intentado explicar desde un punto de vista epistemológico qué hacía posible que existiera conocimiento universal y necesario como la física y las matemáticas. Esto significaba en su perspectiva, explicar cómo eran posible la física newtoniana y la geometría euclideana. Su explicación consistió en mostrar que ambas disciplinas hunden sus raíces en la estructura cognitiva del sujeto trascendental, la física en la facultad del entendimiento y sus herramientas, los conceptos puros o categorías, las matemáticas, en la facultad de la sensibilidad pura, en sus intuiciones puras de espacio y tiempo.

Esta explicación del factum del conocimiento había comenzado a desmoronarse en el siglo XIX como producto del surgimiento de las geometrías no-euclideanas, pero se concretó en la revolución física llevada a cabo por Einstein en la medida en que probaba la aplicación empírica de dichos resultados.

Pero cabe la pregunta, ¿llevó esta revolución a una manera empirista y antikantiana de ver el conocimiento científico? La respuesta es no. Los filósofos antes mencionados se imbricaron en una tarea de reforma del paradigma kantiano, ya iniciada por Helmholtz y Poincaré, que condujo a una manera sorprendentemente moderna de ver la ciencia y sus resultados, y en franca correspondencia con el espíritu de la filosofía kantiana.

Sugeriré que tanto los puntos de vista de Schlick y Reichenbach, quienes abrazaron concepciones de cuño convencionalista, como el del propio Cassirer, quien se mantuvo más fiel a la ortodoxia kantiana, y los cuales pueden verse como desarrollos de las concepciones de Helmholtz y Poincaré, estuvieron comprometidos con un modo de ver la nueva teoría física que apelaba a la existencia de principios a priori constitutivos del objeto de experiencia. 


\section{Convención y coordinación en la nueva teoría física: la propuesta de Schlick}

Tanto en "Die philosophische Bedeutung des Relativitätsprinzips"3 de 1915 -el primer trabajo filosófico publicado sobre la teoría de la relatividad especial-, como en su amplia monografía de 1917 "Raum und Zeit in der gegenwärtigen Physik" "M. Schlick se propone evaluar el impacto profundo que el nuevo marco de principios establecido por Einstein representaba para nuestras intuiciones más arraigadas, y asimismo darle un lugar apropiado dentro de la epistemología.

Hay al menos dos de entre las concepciones más profundamente arraigadas en nuestra cognición que, según Schlick, el principio de la relatividad ${ }^{5}$ viene a impactar, a saber, nuestra visión acerca del tiempo y nuestro concepto de sustancia.

Como es ampliamente conocido, la noción de tiempo absoluto había jugado, en la física newtoniana y aun en la mecánica de Lorentz, el papel de una presuposición incuestionable y se consideraba autoevidente. Por el contrario, la teoría de la relatividad asume que ninguna determinación temporal tiene significación absoluta, sino antes bien que si uno y el mismo proceso se relaciona a diferentes y legítimos sistemas de referencia, es también temporalmente ordenado en diferentes formas. Dos eventos espacialmente separados que para un sistema son simultáneos, se presentan en momentos diferentes para un segundo e igualmente legítimo sistema de referencia. Uno y el mismo proceso, tal como la oscilación del péndulo de un reloj, tiene una duración menor en un sistema en reposo relativo al reloj, que cuando es visto desde un sistema en movimiento relativo al mismo.

Para explicar este cambio en la noción del tiempo, Schlick introduce un movimiento filosófico digno de atención. Según Schlick, la concepción relativista del tiempo no contradice nuestra conciencia inmediata del mismo "for the simple reason that the latter tells us nothing whatever about these properties of time that are dealt with in relativity theory". ${ }^{6}$

${ }^{3}$ Moritz Schlick, "The Philosophical Significance of Relativity", en H. Mulder y F. B. Barbara van de Velde-Schlick, Philosophical Papers (1909-1922). Dordrecht, Reidel, vol. I, 1979.

${ }^{4}$ Versión inglesa de la cuarta edición del año 1922 "Space and Time in Contemporary Physics. An Introduction to the Theory of Relativity and Gravitation" en el volumen citado en la nota anterior.

${ }^{5}$ Schlick distingue entre el principio de la relatividad que enuncia como: "All rectilinear and uniform motions referred to in natural laws are relative" (M. Schlick, $o p$. cit., p.156), y que ve como una ley experimentalmente establecida, y la teoría de la relatividad, i. e., el complejo de conclusiones extraídas por Einstein de su principio.

${ }^{6}$ M. Schlick, op. cit., p. 162. 
El tiempo con el que tratamos en nuestra intuición es, según Schlick, un tiempo psicológico, esto es, algo puramente cualitativo y por lo tanto no medible, mientras que la teoría de Einstein trata específicamente con la medida del tiempo. Veamos esto con algo de detalle.

Haciéndose eco de una afirmación de Kant de los Primeros principios metafísicos de la ciencia de la naturaleza ${ }^{7}$ que reza: "Afirmo, pues, que en toda teoría particular de la naturaleza no podrá encontrarse ciencia en sentido propio, más que en la medida en que pueda encontrarse matemática en ella", ${ }^{8}$ Schlick sostiene que si la ciencia busca someter a todos los fenómenos bajo leyes precisas, éstas han de encontrar expresión únicamente a través de la formulación matemática.

Esta formulación matemática de los fenómenos sólo es posible en la medida en que las regularidades son presentadas en una forma espacio-temporal. Porque es sólo en este modo de presentación, dado que permite la medida y posibilita la obtención de exactitud, por lo que el método matemático es naturalmente aplicable a cantidades medibles.

Ahora bien, en su intento por subsumir todo el orden de los fenómenos a determinaciones numéricas, la ciencia se enfrenta con ciertos dominios que por su naturaleza cualitativa no pueden ser reducidos a meras relaciones cuantitativas.

Entre esos dominios que "escapan" al método matemático propio de la ciencia, ${ }^{9}$ se encuentra el ámbito de lo psicológico, esto es, el dominio de las intuiciones meramente cualitativas, no aprehensibles conceptual o numéricamente. Dentro de este ámbito de lo psicológico se encuentra, a su vez, nuestra conciencia intuitiva del tiempo. Esta conciencia subjetiva del tiempo, debido a sus rasgos cambiantes y accidentales, no permite establecer una definición objetiva del tiempo ni ser medida o usada para medir.

Todo el mundo ha experimentado alguna vez, escuchando una conferencia tediosa, que el tiempo se alarga indefinidamente, o, a la inversa, que una situación agradable pasa frente a nosotros como un flash. Así, sobre esta apre-

${ }^{7}$ Immanuel Kant, Primeros principios de la ciencia de la naturaleza. México, UNAM, 1993.

${ }^{8}$ Ibid., p. 102.

${ }^{9}$ Schlick propone distinguir la ciencia de la filosofía de acuerdo al método de formación de conceptos que cada una de ellas emplea. La primera utiliza el método matemático y persigue la reducción de la mayor cantidad de cualidades posibles a relaciones puramente cuantitativas; la segunda, en tanto, se ocupa de la sistematización del dominio de cualidades puras no susceptibles de reducción a determinaciones matemáticas. Este tema se encuentra desarrollado ampliamente en su artículo "The Boundaries of Scientific and Philosophical Concept-Formation" de 1910 e incluido en los Philosophical Papers, vol. 1 que hemos citado. 
hensión tan cambiante y contingente no podemos esperar erigir conceptos como el de simultaneidad, no al menos si deseamos darle a este tipo de nociones un estatus matemático exacto.

Asimismo, de acuerdo con Schlick, tampoco podemos conceder que proposiciones del tipo de "la duración de un proceso debe ser algo absoluto e independiente de un marco de referencia", tengan su origen en la intuición. Dicho sea de paso, Schlick no niega que ese tipo de proposiciones encierren presuposiciones elementales que "the experience has never till now required us to correct", ${ }^{10}$ pero su estatus está justificado por una vía completamente diferente. Volveré a este punto más adelante.

De este modo, parece que Kant atribuyó propiedades a las formas puras de la intuición que quizás fueran mejor vistas como la contribución del entendimiento o la reflexión. Todo lo cuantitativo, todo lo matemático, y todas las propiedades de la medida temporal deben ser vistas como una contribución del entendimiento y no de las formas subjetivas a priori de la intuición, que constituyen únicamente un ámbito de propiedades puramente cualitativas. ${ }^{11}$

Por otro lado, el tiempo con el que trata la relatividad tiene que ver con la comparación de movimientos y otros procesos. De acuerdo con la teoría de la relatividad, debemos seleccionar una medida del tiempo que depende del estado de movimiento del sistema de referencia. El tiempo "físico" no es algo que pueda ser medido directamente y por lo tanto distinguido de una mera idea, sino que siempre es una "mera idea" que constituye la base de las medidas. Las cantidades $t$ que ocurren en las ecuaciones de la teoría de la relatividad son tiempos "puros, matemáticos" en el completo sentido de la palabra, i.e., son cantidades carentes de elementos cualitativos y por ello medidas con exactitud, es decir, comparadas con una unidad, aunque no sean tiempos absolutos. El tiempo físico es siempre medido y así es una cantidad matemática, una idea. Su opuesto consiste en un tiempo verdaderamente intuitivo o psicológico, pero únicamente real en la conciencia y no susceptible de ser medido, es una cualidad pura.

Como decíamos hace un momento, el segundo de los conceptos que el principio de la relatividad venía a contradecir esencialmente es el concepto de sustancia. El concepto de sustancia, como el de espacio y tiempo, se halla impregnado de ideas filosóficas, pues representa la expresión más general del

${ }^{10}$ M. Schlick, op. cit., p. 163.

${ }^{11}$ Parece bastante claro que Schlick o bien malentendió a Kant o está haciendo una caricatura del kantianismo con el fin de justificar su argumento, pues nadie que haya estudiado mínimamente a Kant puede interpretar la intuición pura en sentido psicológico. Para apreciar un ataque más frontal a la idea de intuición véase especialmente "Is there Intuitive Knowledge?" de 1913, en M. Schlick, op. cit. 
concepto de "ser" de la física. Se entiende por sustancia sencillamente aquel "ser" que constituye la base de todo fenómeno; propiedades y leyes no son más que relaciones entre elementos, a los que corresponde el carácter de sustancia.

La hipótesis del éter venía a expresar este complejo de ideas asociadas con la noción de sustancia, y se propuso esencialmente para suministrar un medio para el fenómeno de la luz, un medio en el cual esta última se propaga a una velocidad de 186000000 de metros por segundo. De acuerdo a las presuposiciones de la teoría de la relatividad, este éter habría de estar en reposo en todo sistema de referencia legítimo, lo que es una contradicción. Porque un cuerpo en reposo en un sistema $K$ está en movimiento relativo a todos los sistemas que se mueven con respecto a $K$, y no puede también estar en reposo en ellos. En la medida en que ningún sistema de referencia es prescrito, puede ser atribuido a cualquier cuerpo físico un movimiento dado, pero tan pronto como se establece tal sistema, ese cuerpo adquiere una velocidad específica relativa a tal sistema: no puede haber éter, debido a que en uno y el mismo sistema habría un número dado de velocidades al mismo tiempo.

Uno puede, no obstante, escoger un sistema legítimo de referencia y afirmar que en ese sistema el éter ha de estar en reposo, pero tal cosa sería completamente arbitraria; de acuerdo con la teoría de la relatividad no habría razones físicas para afirmar tal cosa. No tiene sentido asumir la existencia de un cuerpo físico del cual no puede decirse algo por medios físicos. De este modo, la hipótesis del éter no sirve a los propósitos para los cuales fue propuesta: el éter no es el "medio" para los fenómenos electromagnéticos, porque éstos tienen lugar independientemente de su estado de movimiento.

La filosofía natural, según Schlick, aventajó a la física en la forma en que rechazó desde hace largo tiempo el concepto de sustancia. Ya Hume, Berkeley, y aun Kant rechazaron la idea de dotar a la sustancia de una existencia metafísica (aunque Kant sustituyó su estatus metafísico por uno epistemológico). Sin embargo, podría decirse que la tenacidad con la que los físicos se han adherido a la idea de sustancia proviene de la simple creencia en que la sustancia material es, después de todo, el dominio de la física, y no por una supuesta imposibilidad inherente a su propio modo de conocer, diferente al de la filosofía.

Si recogemos en toda su magnitud las consecuencias que se siguen del establecimiento del principio de la relatividad, esencialmente su ruptura con una concepción que veía a las presuposiciones de la ciencia física intrínsecamente relacionadas con nuestras formas a priori de la intuición, entonces nos encontramos con el hecho sorprendente de que existen esquemas explicativos igualmente consistentes con nuestras intuiciones y con los hechos experimentales, pero incompatibles entre sí. ¿Por cuál de ellos hemos de decidir- 
nos, si todos llevan a cabo coordinaciones aceptables y unívocas con los hechos relevantes que pretenden explicar?

De acuerdo con Schlick, un sistema de conocimiento consiste en un sistema de signos coordinados con los hechos de la realidad, ${ }^{12}$ y si el sistema consigue establecer una coordinación unívoca con esos hechos (sea esto una cosa trascendente o lo inmediatamente dado), entonces el sistema puede llamarse "verdadero". De esta forma, "under certain conditions several theories may be true at once, in that they provide a different but in each case perfectly univocal designation of the facts". ${ }^{13}$

Si tomamos, por ejemplo, el contraste entre los sistemas copernicano y ptolomeico del mundo, según Schlick, no hay experiencia, estrictamente hablando, que pueda probar que el sistema copernicano es el único verdadero; todo lo que la experiencia prueba es que este sistema nos permite asumir las leyes de la mecánica, tal como la ley de inercia, como universalmente válida de una forma perfectamente simple. Las observaciones sólo muestran que el sistema de referencia usado por Copérnico (fijado en el centro del Sol), es preferido sobre el sistema ptolomeico, que es fijado con respecto a la Tierra, en la medida en que aquél conduce a leyes incomparablemente más simples.

En este punto, Schlick invoca la autoridad de Poincaré para defender la tesis de que ninguna experiencia puede obligarnos a establecer un sistema teórico particular, como base para describir las regularidades del mundo físico. Pueden ser elegidos para este propósito innumerables sistemas, con mayor o menor grado de complejidad, pero la elección es siempre producto de una convención. No obstante, apunta Schlick, si bien en principio se trata de una libre elección, de hecho esto nunca opera así, sino que se siguen buenas razones para ello. Se elegirá la más simple, la que conlleve el menor número de hipótesis arbitrarias y esto conduce a Schlick, que era un einsteiniano convencido, a que quizás las teorías más simples estén más cerca de la realidad después de todo, ya que en principio la física presupone la simplicidad de la naturaleza. Parafraseando la máxima de Occam, dice Schlick: "principia non sunt augmenda praeter necessitatem".

${ }^{12}$ La teoría de la coordinación (Zuordnung) a la que se hace referencia aquí fue desarrollada por Schlick en su monumental Allgemeine Erkenntnislehere de 1918. Para una exposición detallada de este punto de vista en comparación con la propia visión de Reichenbach de la coordinación, véase Álvaro Peláez, "Cognición como coordinación en las epistemologías tempranas de M. Schlick y H. Reichenbach”, en Ágora. Papeles de Filosofía, usc, 2005.

${ }^{13}$ M. Schlick, op. cit., p. 168. 


\section{La relatividad y sus "principios coordinativos" a priori}

En su libro de 1920 Relativitätstheorie und Erkenntnis A Priori, ${ }^{14}$ Reichenbach es perfectamente consciente de que los resultados de la teoría de la relatividad afectan decisivamente los principios fundamentales del sistema kantiano del conocimiento -que intentaba avenirse filosóficamente con la física de Newton- y en consecuencia cree que parte de la doctrina kantiana ha de ser abandonada; sin embargo, también cree que la concepción kantiana del conocimiento posee un aspecto que aún puede ser preservado y servir como inspiración para la explicación de la estructura y función de las teorías científicas.

En opinión de Reichenbach, el concepto de a priori en Kant tiene dos diferentes significados, "First, it means 'necessarily truth' or 'true for all times', and secondly, 'constituting the concept of object'”. ${ }^{15}$ De acuerdo a su punto de vista, el primer sentido de lo a priori debe ser rechazado mientras el segundo retenido, lo que lo conduce a sostener que a pesar de que la teoría de la relatividad ha mostrado que los principios de la física newtoniana son insostenibles, no por ello debe asumirse que dicha teoría funciona de una manera meramente empírica, es decir, valiéndose de meras generalizaciones inductivas. Por el contrario, la teoría de la relatividad postula sus propios principios a priori, a los cuales Reichenbach llama "principios coordinativos". ${ }^{16}$

En opinión de Reichenbach, los principios coordinativos a priori son constitutivos del objeto de experiencia en la medida en que conectan las relaciones del ámbito conceptual con lo dado en la experiencia, lo cual en su perspectiva conforma un conglomerado de sensaciones indiferenciadas. Asimismo, tal como ha mostrado la teoría de la relatividad, esos principios son susceptibles de ser abandonados. De estas dos premisas se sigue la conclusión de que conjuntamente con los principios coordinativos se lleva a cabo un cambio en el objeto de experiencia. Veamos cómo se produce este cambio en el caso de la teoría de la relatividad.

Según Reichenbach, la física llega a enunciados cuantitativos a través de la investigación de la influencia de factores físicos sobre determinaciones de extensión o intervalos de tiempo; la medida de distancias e intervalos de tiempo son las medidas cuantitativas primarias. El físico afirma la ocurrencia de

${ }^{14}$ Hans Reichenbach, The Theory of Relativity and A Priori Knowledge. Berkeley/ Los Angeles, Universidad de California, 1965.

15 Ibid., p. 48.

${ }^{16}$ Reichenbach asume también una teoría de la cognición como coordinación, la cual como se ha dicho en la nota número doce, fue propuesta originalmente por Schlick, aunque difiere en aspectos sustanciales de la versión de Schlick. Para estas diferencias véase mi trabajo sugerido en la misma nota al pie. 
fuerzas gravitacionales midiendo el tiempo que un cuerpo en caída libre necesita para atravesar ciertas distancias, o mide el aumento de temperatura por medio del cambio en la altura de una columna de mercurio.

Para este propósito deben ser definidos los conceptos de espacio e intervalo de tiempo. Por espacio e intervalo de tiempo, el físico entiende una razón numérica que conecta el intervalo a ser medido con uno usado como unidad. En esas operaciones, la física tradicional hace la presuposición fundamental de que las extensiones y los tiempos son independientes y que el tiempo definido para un sistema no tiene influencia sobre los resultados de la medida de las extensiones.

En orden a efectuar la transición de las extensiones medidas a relaciones entre ellas, debe ser agregado un sistema de reglas para su conexión. En la física tradicional los teoremas de la geometría euclideana sirven a este propósito, esto es, constituyen asunciones imprescindibles para medir cualquier cambio en la extensión o en el tiempo. Se ve como una propiedad necesaria de los cuerpos físicos el que se comporten de acuerdo a esas relaciones generales, esto es, sólo bajo esta presuposición puede algo ser pensado como una cosa física.

Obtener conocimiento cuantitativo no significa otra cosa que aplicar esas reglas generales a la realidad y ordenar los valores numéricos en un sistema. Esas reglas pertenecen al concepto del objeto de la física.

Ahora bien, cuando la teoría de la relatividad cambió esas reglas, surgieron serias dificultades conceptuales. La teoría presupone que las extensiones y los intervalos de tiempo medidos no poseen validez absoluta, sino que contienen elementos que en la concepción anterior se verían como accidentales: el sistema de referencia escogido y el hecho de que un cuerpo en movimiento mostrará una contracción en relación con un sistema en reposo. Este resultado fue interpretado como contradiciendo la causalidad, debido a que no podía ser indicada ninguna causa para tal contracción. Sorpresivamente nos encontramos enfrentados a un cambio físico cuya causa no podía ser reconciliada con ninguna concepción de la fuerza producida por el movimiento. Según Reichenbach, "...the apparent difficulty does not arise from the attempt to preserve the requirement of causality, but from the attempt to preserve a concept of object that the theory of relativity has overcome".${ }^{17}$

De acuerdo con Reichenbach, "There exists a definite cause for the contraction of length: the relative motion of the two bodies. Depending on which system of reference is assumed to be at rest, either of the two bodies can be called shorter". ${ }^{18}$ Así, si este resultado es interpretado como contradiciendo

${ }^{17}$ H. Reichenbach, op. cit., p. 96.

${ }^{18}$ Idem. 
la causalidad, debido a que ésta requiere la afirmación de por qué el cuerpo "realmente" se contrae, se asume luego que la extensión es una propiedad absoluta de los cuerpos. Pero Einstein ha mostrado que la extensión es una magnitud definida sólo con relación a un cierto sistema de coordenadas.

Entre un cuerpo en movimiento y una barra de medir existe una relación; pero dependiendo del sistema de referencia escogido, esta relación se manifestará como una extensión en reposo, como una contracción de Lorentz, o como una extensión de Lorentz. Lo que medimos como extensión no es la relación entre los cuerpos, sino su proyección en un sistema de coordenadas.

Podemos formular esta extensión sólo en un sistema de coordenadas; pero al indicar simultáneamente las fórmulas de transformación para todo otro sistema, nuestro enunciado obtiene significación objetiva. El nuevo método de la teoría de la relatividad consiste, para Reichenbach, en asignar un significado objetivo a enunciados particulares, mediante la indicación de las fórmulas de transformación. Este método cambia el concepto de relación física. Una extensión medida sólo puede ser establecida y así ser llamada "objetiva" en un sistema específico, pero esta extensión es sólo una expresión de la relación física. Lo que era anteriormente visto como una extensión geométrica no es en absoluto una propiedad de un cuerpo, sino antes bien el reflejo de tal propiedad en la descripción de un sistema simple de coordenadas.

Debemos notar el cambio en el concepto del objeto: lo que era anteriormente visto como una propiedad de las cosas se vuelve ahora una propiedad de las cosas y sus sistemas de referencia. Así, en palabras de Reichenbach, "The physics offorces and things is replaced by the physics of states of fields" ${ }^{19}$

Como es notorio por las anteriores consideraciones, Reichenbach comparte con Schlick la idea de interpretar a la teoría de la relatividad como proporcionando la prueba de que es posible pensar coordinaciones arbitrarias e igualmente consistentes con los datos. El énfasis de Reichenbach, no obstante, recae sobre el poder constitutivo de los principios que coordinan los sistemas de axiomas con los datos de la realidad, posibilitando con su cambio, un verdadero cambio en el objeto de conocimiento empírico. Recuérdese que, según Reichenbach, los principios constitutivos determinan que sea un objeto individualmente considerado, dentro del continuo de la experiencia. Asimismo, si bien en principio Reichenbach se avendría a un modo convencionalista de ver las cosas, desde el cual podría verse a la historia de la ciencia como compuesta de una serie de discontinuidades, de hecho cree que esto no es posible, pues representaría que la ciencia comenzara desde cero cada vez.

${ }^{19}$ Ibid., p. 103. El énfasis es de Reichenbach. 
En su lugar propone el método de las aproximaciones sucesivas, el cual lo aleja del convencionalismo hacia formas más tradicionales de ver el desarrollo de la ciencia, con un énfasis importante en el principio de inducción normal.

\section{La covariancia general y la unidad de la razón: la propuesta de E. Cassirer}

La tarea que enfrenta Cassirer en su monografía de 1921 Zur Einsteinschen Relativitätstheorie. Erkenntnistheoretische Betrachtungen, ${ }^{20}$ es entender cabalmente qué quiere decir Einstein cuando afirma de su teoría que mediante ella "the last remainder of physical objectivity is taken from space and time". ${ }^{21}$ La pregunta que se hace Cassirer respecto de esa observación de Einstein es: "What are we to understand by the physical objectivity, which is here denied to the concepts of space and time?"22

De acuerdo con Cassirer, el físico puede percatarse de que el espacio y el tiempo no llenan el requisito de Planck de que "todo lo que puede ser medido existe", pero el epistemólogo investiga el establecimiento de esos estándares de medida, los cuales cambian como resultado de diferentes puntos de vista de conocimiento. De acuerdo a este cambio en el "ideal standpoint" surgen para el pensamiento, distintas clases y sistemas de objetos, todos definidos por la forma del juicio característico de donde proceden.

Es claro que esta visión de la constitución de los objetos no coincide en modo alguno con la manera realista ingenua que los ve como realidades independientes dados a la percepción sensorial. La epistemología revela que lo que la visión ingenua toma como un hecho extraído de la experiencia sólo responde a un conjunto de hipótesis, principios, o axiomas que son fijados con anterioridad a toda experiencia. Quizás entonces, la afirmación de Einstein que niega objetividad al espacio y al tiempo, "mean something else and something deeper than the knowledge that the two are not things in the sense of naive realism". ${ }^{23}$

En opinión de Cassirer, lo que debe ser investigado es la posición lógica especial de los conceptos de espacio y tiempo, los que a pesar de compartir con el resto de los conceptos físicos su estatus como conceptos de medida,

${ }^{20}$ Ernst Cassirer, Substance and Function and Einstein's Theory of Relativity. Trad. de W. Swabey y M. Swabey. Chicago, Open Court, 1923.

${ }^{21}$ Apud E. Cassirer, op. cit., p. 356.

${ }^{22}$ E. Cassirer, op. cit., p. 356.

${ }^{23}$ Apud ibid., p. 357. 
son vistos como "concepts and forms of measurement of an order higher than the first order". ${ }^{24}$ Así, cualquier intento de dar una respuesta a la cuestión concerniente a la pérdida de "objetividad física" de los conceptos de espacio y tiempo, está restringida a reconocer el carácter más fundamental de esos conceptos. De acuerdo con Cassirer, la respuesta debe ser buscada en términos de las manifestaciones cambiantes del concepto de objeto físico dentro de la ciencia física. De este modo, la tarea específica que Cassirer se ha impuesto a sí mismo es un examen de cómo ha de ser construida la "objetividad física" desde dentro de la nueva perspectiva, tal que ésta es negada al espacio y al tiempo.

Ya en la discusión del capítulo II sobre los fundamentos conceptuales y empíricos de la teoría de la relatividad, Cassirer llega a un resultado preliminar: el requerimiento de covariancia general, a saber, el que las leyes son establecidas en una forma válida para todos los marcos de referencia, representa un tipo de avance similar al que ocurrió en la transición desde la mecánica newtoniana a la teoría especial de la relatividad. En este último caso, la validez de las leyes generales de la naturaleza estaba restringida en relación con una determinada clase de cuerpos de referencia; con la relatividad general esta restricción desaparece. Y aunque algún sistema de referencia está implicado en la contrastación de esas leyes, su significado y valor es independiente de la particularidad de esos sistemas y "remains indentical with itself, whatever changes experiences may bring to them". Es precisamente esta independencia del punto de vista del observador que está implicada cuando hablamos de un "objeto de la naturaleza" y de "leyes de la naturaleza" como determinadas en sí mismas. Sólo en este estadio de la investigación física podemos afirmar, según Cassirer, que "the anthropomorphism of the natural sensuous picture of the world, the overcoming of which is the real task of physical knowledge, is here again forced a sep further back" ${ }^{25}$

La interpretación de la covariancia general como un desarrollo del principio metodológico de la "unidad objetivadora" es reiterada a lo largo de todo el ensayo de Cassirer.

La teoría general de la relatividad adopta el principio de que para la descripción física de los procesos de la naturaleza, ningún cuerpo de referencia debe ser distinguido de otro. Este principio es entendido por Cassirer como una "regla del entendimiento" hipotéticamente adoptada dentro de la física. El único significado y justificación de esta norma descansa en el hecho de que, a través de su aplicación, es posible obtener la unidad sintética de los fenómenos en sus relaciones temporales, esto es, la explicación legaliforme de los hechos observados.

\footnotetext{
${ }^{24}$ Ibid., p. 358.

${ }^{25}$ Ibid., pp. 381-382.
} 
Cassirer advierte que quizás se podría estar tentado a entender el principio de covariancia general como una afirmación analítica, que se sigue del significado que atribuimos al concepto de "ley", sin embargo, afirma con convicción, "the demand, that there must in general be such ultimate invariants, is synthetic". 26

El principio de covariancia general es una máxima suprema establecida para la investigación de la naturaleza, es una restricción formal, pero también, de acuerdo con Cassirer (citando a Einstein), es "a heuristic aid in the search for the general laws of nature". ${ }^{27}$

Cuando llegamos al capítulo v de la monografía, el que trata específicamente de los conceptos de tiempo y espacio, nos encontramos con un tratamiento especial de lo que habitualmente se había visto (y se ve), como el obstáculo más importante para la concepción kantiana de la física, el problema de la intuición pura. Sorprendentemente, Cassirer sostiene que la teoría general de la relatividad no sólo no contradice el sentido de la intuición pura, sino que lo mejora clarificando el rol que ésta juega en la cognición empírica. ${ }^{28}$

Interpretando a Kant de manera poco convencional, Cassirer dice que el espacio y el tiempo son fuentes conceptuales de conocimiento, conceptos ideales puros de los órdenes de coexistencia y sucesión. ${ }^{29}$ En este sentido, la teoría de la relatividad, al quitarle a la intuición pura su naturaleza sensible, ha clarificado el sentido kantiano del término, cuyo significado "was not always grasped by Kant with equal sharpness, since more special meanings and applications were substituted involuntary in his case". ${ }^{30}$ De acuerdo con Cassirer, la intención de Kant era plasmar en una idea la naturaleza de la forma serial de coexistencia y sucesión; pero estas formas no han de ser concebidas como rígidas, sino "living and moving". Ninguna de ellas puede ser explicitada de un solo golpe, sino a través de un proceso imbricado con las manifestaciones del pensamiento científico. Y la tarea de la ciencia física, que se encuentra en medio de lo sensible y lo formal puro, es la de relacionar progresivamente ambos dominios, y de esta manera lo sensible "increasingly loses its 'contingent' anthropomorfic character and assumes the imprint of thought, the imprint of systematic unity of form". ${ }^{31}$

${ }^{26}$ Ibid., p. 384.

${ }^{27}$ Apud ibid., p. 377. El énfasis es de Cassirer.

${ }^{28}$ Adviértase la contraposición con la visión de Schlick examinada más arriba.

${ }^{29}$ En realidad esto se parece más a la concepción de Leibniz que a la de Kant, pues recuérdese que este último insiste una y otra vez en que el espacio y el tiempo son una fuente de conocimiento esencialmente no-conceptual.

${ }^{30}$ E. Cassirer, op. cit., p. 418.

${ }^{31}$ Ibid., p. 421. 
La pérdida de "objetividad física" del espacio y el tiempo, triunfalmente anunciada por Einstein, encuentra su manifestación fundamental en la filosofía crítica a través del reconocimiento de que espacio y tiempo son sólo formas funcionales de sucesión y coexistencia.

\section{Conclusiones}

Como es más o menos conocido, los filósofos convencionalistas de finales del siglo XIX y comienzos del XX -entre ellos, Henri Poincaré, Pierre Duhem y E. Le Roy- iniciaron, principalmente a la luz de los desarrollos en la geometría, una labor de reconstrucción de la epistemología de cuño kantiano. En 1898, Henri Poincaré desarrolló una concepción acerca del origen de la noción de espacio de claro corte kantiano. En efecto, en su opinión "Nos sensations ne peuvent pas nous donner la notion d' espace. Cette notion est construite par l'esprit avec des éléments qui preexistent en lui, et l'experience externe n'est por lui que l'occasion d'exercer ce pouvoir..."32

Como ya había sido adelantado por Helmholtz, Poincaré cree que el origen matemático y la aplicación empírica de la geometría matemática ha de ser explicada mediante la estructura del grupo de movimientos rígidos del sujeto percipiente en el espacio perceptual, expresada en la condición de movilidad libre. Poincaré coincide también en que la estructura de este grupo (i.e. la estructura del espacio perceptual) está basada en nuestras sensaciones motoras o corporales en la medida en que movemos o desplazamos nuestro cuerpo en y a través del espacio perceptual. Finalmente, Poincaré también coincide con Helmholtz en que debido a que la condición de movilidad libre da lugar a cualquiera de los tres casos de geometrías clásicas de curvatura constante, la geometría específicamente euclideana no es una necesidad del pensamiento ni un producto de nuestra forma de intuición a priori. Asimismo, dado que nuestra experiencia sensible no puede arrojarnos, debido a su inherente ambigüedad, una estructura u otra, el grupo con el que culminamos debe reflejar inevitablemente nuestra libre elección. Elección que es guiada pero no restringida por esa experiencia tosca y aproximada.

Esto nos conduce a un aspecto interesante de la concepción de Poincaré, que a menudo es pasado por alto, ${ }^{33}$ y que aparece en la cita consignada antes.

${ }^{32}$ Henri Poincaré, "Des Fondements de la Geometrie" [1898], en Laurent Rollet, ed., Scientific Opportunism: An Anthology. Basel, Birkhauser, 2002, p. 5.

${ }^{33}$ Friedman ha tratado brillantemente la relación entre Kant, Helmholtz y Poincaré, aunque se ha centrado en la importancia que esos autores asignan a la intuición en la noción de espacio. A diferencia de esto mi énfasis está en que en Poincaré se gesta una 
Como bien decíamos, nuestra experiencia sensible y la reflexión sobre ella, nos sugiere determinadas estructuras geométricas que nunca coinciden plenamente con sus respectivas formulaciones matemáticas.

Estas inferencias, no obstante, no proceden de una forma meramente empírica. Si bien la geometría puede ser definida como el estudio del movimiento de los sólidos, es claro que no se ocupa en realidad de los sólidos naturales, sino que tiene por objeto ciertos sólidos ideales, absolutamente invariables, que no son más que una imagen simplificada y bastante lejana de aquellos. La noción de esos cuerpos ideales es, según Poincaré, extraída completamente de nuestro espíritu, y la experiencia sólo es una ocasión que nos ayuda a hacerla surgir. Aún más, la investigación de los grupos de movimientos de cuerpos rígidos sólo es posible porque "le concept général de groupe préexiste dans notre esprit au moins en puissance". ${ }^{34}$ Es decir, se nos impone como una forma de la facultad del entendimiento.

Esta idea de Poincaré, que ya había estado también en el centro de la concepción de Helmholtz, ${ }^{35}$ tiene la ventaja extra de que a pesar de que la noción de "grupo" condiciona la formación de la noción de espacio deja, no obstante, en la medida en que se reconoce que dicha noción que así surge no posee la precisión y exactitud que caracteriza a las formulaciones matemáticas, lugar a la libre elección.

La filosofía posterior reaccionó a esta concepción de dos maneras bien definidas. Unos, entre los cuales podemos contar a Schlick y Reichenbach, abandonaron toda apelación a la idea de la existencia de determinadas nociones que se encuentran atrincheradas en nuestro aparato cognitivo. Otros, entre los cuales contamos a los miembros de la escuela neokantiana y principalmente a Cassirer, se mantuvieron fieles a este aspecto, aunque dándole un nuevo contenido a la luz de los avances en la lógica formal de principios de siglo. Sin embargo, ambos se mantuvieron fieles al aspecto fundamental de la idea en cuestión, a saber, la naturaleza constitutiva de esas nociones o principios. Schlick y Reichenbach, a pesar de sus diferencias, se orientaron hacia formas más o menos

idea de entendimiento y de constitución de la experiencia que obvia el recurso a la intuición y actúa bajo la forma de "idealizaciones" (Michael Friedman, "Geometry, Construction and Intuition in Kant and his Successors", en G. Sher y R. Tieszen, eds., Between Logic and Intuition: Essays in Honor of Charles Parsons. Cambridge, Universidad de Cambridge, 2000).

${ }^{34}$ H. Poincaré, op. cit., p. 90.

${ }^{35}$ Helmholtz expresa este punto de vista en el título mismo de uno de los apéndice a su trabajo más conocido "Die Tatsachen in der Wahrnemung" de 1878, como sigue: "El espacio puede ser trascendental sin que los axiomas lo sean" (H. Helmholtz, "The Facts in Perception", en R. Cohen y Y. Elkana, eds., Hermann von Helmholtz: Epistemological Writings. Holanda, Reidel, 1977). 
convencionalistas de ver las cosas. El primero más abiertamente, en la medida en que renuncia a toda constricción a priori en la elección de dichos principios. El segundo, aunque también abandona la idea de que los diferentes marcos conceptuales se impongan como una necesidad de la razón, no obstante concede al "principio de inducción normal", el que considera como un principio $a$ priori, la tarea de orientar la decisión por uno u otro marco conceptual. Lamentablemente, sus consideraciones sobre el estatus de este principio son irremediablemente oscuras, arrojándolo al limbo de lo "ultimo, inanalizable".

Cassirer, por su parte, recoge las intuiciones de Helmholtz y Poincaré acerca del rol constitutivo de ciertas nociones formales que pertenecen a nuestra facultad de entendimiento, pero recogiendo al mismo tiempo los resultados de la lógica formal, entiende esa noción como una función lógica generadora de series que coincide en sus características generales con la moderna noción matemática de función, la cual es la fuente única de generación conceptual tanto en las matemáticas como en la ciencia empírica.

De este modo, para Schlick, la teoría de la relatividad establece un nuevo marco conceptual que permite una coordinación unívoca con los hechos de la experiencia que es incomparablemente más simple que las coordinaciones anteriores. Sus determinaciones puramente matemáticas excluyen cualquier aspecto cualitativo o psicológico en el conocimiento, lo cual es relegado al nivel de la experiencia subjetiva. Así entiende Schlick, la demanda de Einstein de que espacio y tiempo deben ser "limpiados" de cualquier elemento subjetivo. Reichenbach, como ha sido dicho, también parte de consideraciones convencionales acerca del papel de la pluralidad de coordinaciones unívocas con la realidad. Sin embargo, su énfasis en el método de las aproximaciones sucesivas lo aleja de consideraciones puramente arbitrarias en lo tocante a la construcción de sistemas conceptuales. La teoría de la relatividad es vista como un claro ejemplo de un cambio "natural" en el concepto del objeto de conocimiento físico. Cassirer, finalmente, hace ver que en el tratamiento por parte del idealismo crítico de las nociones de espacio y tiempo se halla cumplido el desideratum de Einstein respecto de la desantropomorfización de esas nociones. Espacio y tiempo son, para Cassirer, esos invariantes últimos que proveen la forma de la coexistencia y la sucesión de los objetos de la experiencia, pero esas estructuras formales no son dadas de una vez y para siempre, sino que sus determinaciones son dependientes de las manifestaciones conceptuales que se proponen a lo largo de la historia de la física. De esto se sigue, que no es posible decir qué cosas sean esos invariantes, más que lo dicho recientemente, formas conceptuales que determinan la coexistencia y la sucesión de los fenómenos de la naturaleza.

Asimismo, de crucial importancia es su tratamiento del principio de covariancia general, el que ve como un principio a priori del entendimiento 
que posibilita la unidad explicativa de las leyes de la naturaleza. Quizás deberíamos apuntar que también en este punto Cassirer se aleja un paso más de la ortodoxia kantiana, en la medida en que parece dotar de un valor constitutivo a principios que tienen un papel esencialmente heurístico y que Kant ve como principios meramente regulativos. Sin embargo, los desarrollos en las ciencias formales y naturales explican el tránsito desde la concepción kantiana original a las formulaciones de Cassirer.

De este modo, vemos cómo la filosofía de la ciencia de comienzos del siglo $\mathrm{xx}$, la cual puede constituirse como ejemplo de la relación que la epistemología ha de guardar con la ciencia empírica, arriba, a través de la asunción consciente de los errores y problemas de la visión kantiana original, a una concepción de la estructura y función de las teorías científicas que hace justicia tanto a las profundas intuiciones que Kant tuvo respecto del modo de operar de la ciencia de su época, como a los propios desarrollos a los que las disciplinas científicas, formales y empíricas, habían conducido. Quizás Kant se equivocó al decir que el marco conceptual newtoniano era universal y necesario, pero, según parece, la práctica científica que él intentó caracterizar, no parece haber cambiado muy radicalmente. 
